\title{
SAR Evaluation of Ultra Wideband (UWB) Textile Antennas
}

\author{
${ }^{1,3}$ P. J. Soh, ${ }^{1}$ G. A. E. Vandenbosch, ${ }^{3}$ F. H. Wee, ${ }^{1}$ M. Mercuri, ${ }^{2}$ A. van den Bosch, ${ }^{2}$ M. Martinez- \\ Vazquez, and ${ }^{1}$ D. M. M.-P. Schreurs \\ ${ }^{1}$ Div. ESAT- TELEMIC, KU Leuven, Kasteelpark Arenberg 10, 3001 Leuven, BELGIUM \\ ${ }^{2}$ IMST GmbH, Carl-Friedrich-Gauss-Str. 2-4-4, 47475 Kamp-Lintfort, GERMANY \\ ${ }^{3}$ School of Computer and Communication Engineering, Universiti Malaysia Perlis (UniMAP), Pauh \\ Putra Campus, 02600 Arau, Perlis, MALAYSIA
}

\begin{abstract}
This work presents a systematic investigation of the Specific Absorption Rate (SAR) of two ultra wideband (UWB) textile antennas. These popular UWB topologies are implemented using similar materials and evaluated at a common frequency point for comparison purposes in a certified SAR facility. Evaluations indicate that the antenna ground plane area and the distance to the body phantom also seriously affects SAR levels. This evaluation has also quantified the SAR measurement repeatability at various distances at a maximum of $10 \%$.
\end{abstract}

Index Terms - Specific Absorption Rate, biomedical applications of electromagnetic radiation, ultra wideband antennas.

\section{INTRODUCTION}

The application of the Wireless Body Area Networks (WBAN) for various medical and non-medical usages such as disability assistance, entertainment, sports, etc. have triggered an extensive research effort into utilizing conformal materials. One of such efforts towards realizing a fully-worn setup is the use of wearable and flexible antennas made from textiles. This option is promising as the applicability of such materials has been successfully demonstrated by previous researchers [1]-[4].

The Ultra Wideband (UWB) physical layer (PHY) is especially interesting, as it is capable of offering robust performance for WBANs, besides implementation opportunities for high performance, robustness, low complexity and ultra low power operations. The UWB operating band is divided into two band groups, low band and high band. Each band is divided into channels, each with a bandwidth (BW) of $499.2 \mathrm{MHz}$. The low band requires a mandatory channel (Channel 1$)$ centered at $f_{\mathrm{c}}=$ 3993.6 MHz, whereas the high band mandatory channel (channel 6) is centered at $f_{\mathrm{c}}=7987.2 \mathrm{MHz}$ [5].

The main challenge to operate such radiating structures in close-proximity to the human user is the variation of their performance parameters - reflection coefficient $\left(S_{11}\right)$, BW, gain, efficiency and radiation characteristics. This is expected to be caused by coupling and absorption by the human body. While these evaluations are influential in determining proper on-body operation, another crucial parameter often investigated with the aid of simulators is its on-body operational safety level, defined as its Specific Absorption Rate (SAR) [6]. Previous SAR literature mostly reported antennas and communication devices made from conventional printed circuit board (PCB) boards operating near the human head or hands [7]-[8].

Two UWB all-textile antennas implemented on a common substrate are selected for SAR evaluation at 5.2 $\mathrm{GHz}$ - their topologies and sizes are chosen to be distinct to better understand their resulting SAR. The evaluation at $5.2 \mathrm{GHz}$ will also possibly provide an idea on the worstcase SAR estimation within the $6 \mathrm{GHz}$ limit specified in the IEC 62209 standard [6] using a certified liquid and experimental setup, as higher SARs are expected at higher frequencies due to the higher tissue conductivities. The antenna topologies and materials will first be introduced prior to the SAR simulation and measurement procedures. The major observations are discussed prior to a findings summary in the conclusion section.

\section{ANTENNA MATERIALS AND TOPOLOGY}

The antenna prototyping materials consist of two textile types, conducting and non-conducting. The former, ShieldIt Super from LessEMF Inc. is used to form the radiator, ground plane and transmission lines for both antennas. It is $0.17 \mathrm{~mm}$ thick $(t)$ with an estimated conductivity of $\sigma=1.18 \times 10^{5} \mathrm{~S} / \mathrm{m}$ [2]-[4]. The nonconductive felt, with $3 \mathrm{~mm}$ thickness, relative dielectric permittivity $\left(\varepsilon_{r}\right)$ of 1.45 and loss tangent $(\tan \delta$ ) of 0.044 is used as the substrate. ShieldIt is secured onto the felt by activating the hot melt on its reverse side at $130^{\circ} \mathrm{C}$. $\mathrm{RF}$ power is fed to the antennas through $50 \Omega$ SMA connectors soldered to ShieldIt textile at a maximum temperature of $200^{\circ} \mathrm{C}$.

Two popular UWB antenna topologies are chosen for evaluation. The first is based on a typical UWB monopole antenna without any rear ground plane, whereas the second UWB antenna includes a slotted rear ground. As 
mentioned earlier, their topologies are chosen to be distinct and tested at a common frequency point, i.e., at $5.2 \mathrm{GHz}$ with at least $-10 \mathrm{~dB}$ of $S_{11}$. Each antenna topology is first described in the following sections.

\section{A. UWB Monopole Antenna without Rear Ground Plane}

The first design is based on a co-planar waveguide (CPW)-fed rectangular monopole, with the ground plane placed on the same layer as the radiator, next to the CPW feeding line [9]. This physically provides no shielding against coupling when mounted on-body, which is very distinct compared to the next structure. The monopole's overall size is defined by its substrate sized at $36 \times 42$ $\mathrm{mm}^{2}$, and this prototype is referred to as plain CPW-fed monopole (PCPWM). Its topology and dimensions are shown in Fig. 1(a)-(b).

\section{B. UWB Wideslot Antenna with Partial Ground Plane}

The second UWB antenna topology is based on a microstrip line-fed radiator on the top layer, with a slotted ground plane on its reverse side. A rectangular parasitic element is centered within the trapezoidal-shaped slot on its ground plane to improve its bandwidth. This antenna, referred to as the Wide Slot UWB antenna (WSUWB) is modified from [10], and has been prototyped to enable evaluation of a topology with a partial rear ground plane and a smaller overall size. This antenna is sized at $29 \times 27$ $\mathrm{mm}^{2}$, and its ground plane and parasitic element covers ca. $70 \%$ of the antenna's reverse side. Its topology and dimensions are shown in Fig. 1(c)-(d).

\section{EVAlUATION SETUP}

For SAR evaluations, a commercial DASY-4 system from Schmid \& Partners Engineering AG (SPEAG) is used to perform the measurements in proximity of a Specific Anthropomorphic Mannequin (SAM). It is filled using a standard tissue-emulating liquid at $5.2 \mathrm{GHz}$, with $\varepsilon_{r 5.2}=36, \sigma_{5.2}=4.66 \mathrm{~S} / \mathrm{m}$. Prior to antenna under test (AUT) measurements, calibrations are performed according to the procedure in [11]. The lineup is first connected to a power meter to ascertain that a $100 \mathrm{~mW}$ constant wave (CW) signal is fed into the AUTs during subsequent evaluations. The AUT is secured onto a positioner below the SAM to ensure a fixed distance during measurement. The AUTs are first placed at varying distances $(d)$ from the phantom, i.e., at 2, 5, 10 and 15 $\mathrm{mm}$. Distances of $d>10 \mathrm{~mm}$ are more realistic in emulating practical situations, e.g., a $5 \mathrm{~mm}$-thick jacket and another (average) $5 \mathrm{~mm}$ air gap allowance for the jacket-to-body distance. These distances (d) are also decided based on the fact that AUTs must be placed with $d$ $<20 \mathrm{~mm}$ on the body trunk for SAR measurements [6].

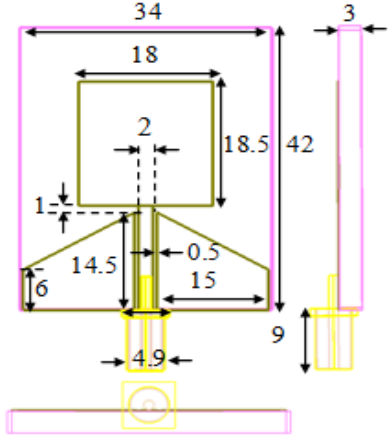

(a)

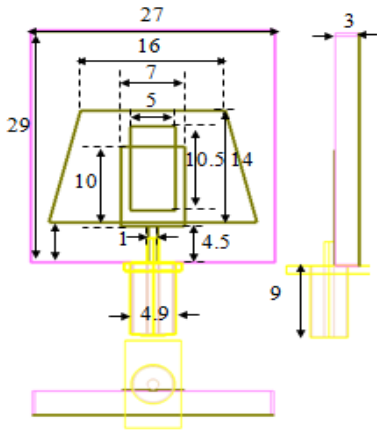

(c) (b)

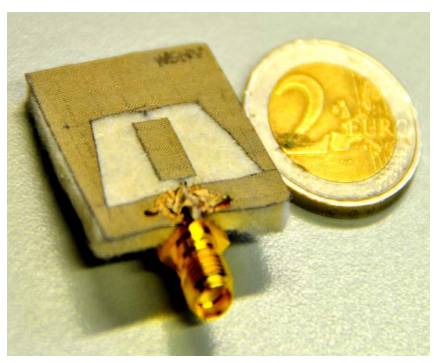

(d)

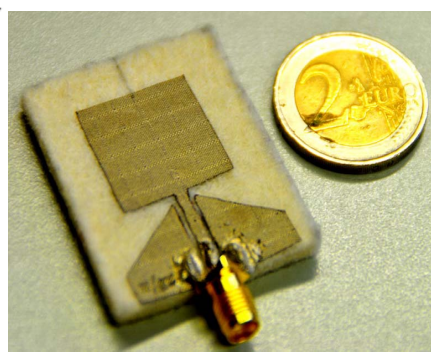

Fig. 1. The UWB antennas: (a) PCPWM (schematic), (b) PCPWM (fabricated), (c) WSUWB (schematic), and (d) WSUWB (fabricated).

\section{RESULTS AND DISCUSSION}

\section{A. Measurement Repeatability}

A good first step in any measurement campaign is to determine repeatability at a specified frequency and AUT distance. For this purpose, PCPWM has been used to evaluate this repeatability at two $d$ 's, i.e., at 5 and $10 \mathrm{~mm}$. The AUT is disconnected and re-connected in between consequent measurements, with the distance between the phantom shell and antenna carefully determined. A maximum uncertainty of $3 \%$ at $d=5 \mathrm{~mm}$ and $10 \%$ at $d=$ $10 \mathrm{~mm}$ was found, as listed in Table I.

\section{B. SAR Evaluation}

Table II summarizes the SAR for PCPWM and WSUWB for $d=2,5,10$ and $15 \mathrm{~mm}$. Results generally indicate that SAR for both antennas increases with decreasing $d$. The existence of the ca. $70 \%$ rear ground plane area in WSUWB also consistently produced lower SAR in comparison to the PCPWM prototype. However, the WSUWB-PCPWM SAR difference varies when evaluated at different $d$. Less than $4 \%$ of SAR difference is observed between the two antenna types when $d=2$ 
$\mathrm{mm}$, and this difference grew larger for $d=10 \mathrm{~mm}$ and $d$ $=15 \mathrm{~mm}$, with $12 \%$ and $18 \%$, respectively. This is partially attributed to the increasing measurement repeatability as $d$ is increased. Note also that both antennas placed at $d<5 \mathrm{~mm}$ exceeded the $2 \mathrm{~W} / \mathrm{kg}$ European SAR limit. Although lower power levels are expected to be fed into such antennas for UWB applications, this evaluation indicated that the choice of antennas without or with partial rear ground planes, which is also a popular technique in enlarging bandwidths, poses a risk in terms of SAR. Thus, careful consideration is needed in selecting the proper UWB antenna topology to avoid SAR levels exceeding the regulatory limit when operating too close to the body.

TABLE I

SUMMARY OF REPEATABILITY TEST USING PCPWM

\begin{tabular}{|l|c|c|}
\hline $\begin{array}{l}\text { Antenna type/ } \\
\text { Run no. }\end{array}$ & Distance (mm) & Meas. SAR (W/kg) \\
\hline PCPWM Run 1 & 5 & 2.78 \\
\hline PCPWM Run 2 & 5 & 2.93 \\
\hline PCPWM Run 1 & 10 & 1.33 \\
\hline PCPWM Run 2 & 10 & 1.33 \\
\hline PCPWM Run 3 & 10 & 1.54 \\
\hline
\end{tabular}

TABLE II

SUMMARY OF SAR WITH VARYING DISTANCES AT $5.2 \mathrm{GHz}$

\begin{tabular}{|c|c|c|c|}
\hline Antenna type & $\begin{array}{c}\text { Distance } \\
(\mathrm{mm})\end{array}$ & $\begin{array}{c}\text { Meas. SAR } \\
(W / k g)\end{array}$ & Diff (\%) \\
\hline PCPWM & 2 & 3.390 & \multirow{2}{*}{3.83} \\
\hline WSUWB & 2 & 3.260 & \\
\hline PCPWM (Ave) & 5 & 2.855 & \multirow{2}{*}{6.13} \\
\hline WSUWB & 5 & 2.680 & \\
\hline PCPWM (Ave) & 10 & 1.400 & \multirow{2}{*}{12.14} \\
\hline WSUWB & 10 & 1.230 & \\
\hline PCPWM & 15 & 0.699 & \multirow{2}{*}{18.03} \\
\hline WSUWB & 15 & 0.573 & \\
\hline
\end{tabular}

\section{CONCLUSION}

A systematic SAR evaluation of two UWB textile antennas with different sizes and topologies at $5.2 \mathrm{GHz}$ is presented. Measurements are performed in a certified, commercial measurement facility using several distances between the antenna and SAM phantom. As expected, the antenna without a rear ground plane produced worse SAR level compared to the antenna with a partial ground plane. Evaluation at different distances from the body also indicated that SAR for both antennas are affected by the placement distance from the body - a higher SAR with a decreasing distance. Measurements are also proven to be reproducible with a maximum repeatability of $10 \%$.

\section{ACKNOWLEDGEMENT}

The authors wish to acknowledge the Malaysian Ministry of Education - Higher Education Sector, the COST action ic1102 VISTA (Versatile, Integrated and Signal-Aware Technologies for Antennas) and the Hercules Foundation for their financial assistance.

\section{REFERENCES}

[1] I. Locher, M. Klemm, T. Kirstein, and G. Troster, "Design and Characterization of Purely Textile Patch Antennas," IEEE Trans. on Advanced Packaging, vol. 29, no. 4, pp. 777-788, 2006.

[2] P. J. Soh, G. A. E. Vandenbosch, S. L. Ooi, and N. H. M. Rais, "Design of a Broadband, All-Textile Slotted PIFA," IEEE Trans. Antennas and Propagation, vol. 60, no. 1, pp. 379-384, Jan. 2012.

[3] P. J. Soh, G. A. E. Vandenbosch, S. L. Ooi, and M. R. N. Husna, "Wearable Dual-Band Sierpinski Fractal PIFA using Conductive Fabric," Electronics Letters, vol. 47, no. 6, pp. 365-367, March 2011.

[4] P. J. Soh, S. J. Boyes, G. A. E. Vandenbosch, Y. Huang, and S. L. Ooi, "On-body Characterization of a Dual-Band, All-Textile PIFA," Prog. in Electromagnetic Research, vol. 129, pp. 517-539, 2012.

[5] Local and Metropolitan Area Networks, Part 15.6: Wireless Body Area Networks, IEEE 802.15.6 Standard, 2012.

[6] Human Exposure to Radio Frequency Fields from Handheld and Body-mounted Wireless Communication Devices Human Models, Instrumentation and Procedures, IEC 62209 Standard, 2010.

[7] A. W. Guy and C.-K. Chou, "Specific Absorption Rates of Energy in Man Models Exposed to Cellular UHF MobileAntenna Fields," IEEE Trans. on Microwave Theory and Techniques, vol. 34, no. 6, pp. 671-680, Jun 1986.

[8] M. Okoniewski and M. A. Stuchly, "A study of the handset antenna and human body interaction," IEEE Trans. on Microwave Theory and Techniques, vol. 44, no. 10, pp. 1855-1864, Oct 1996.

[9] P. J. Soh, G. A. E. Vandenbosch, and J. Higuera-Oro, "Design and Evaluation of Flexible CPW-fed Ultra Wideband (UWB) Textile Antennas", IEEE RF and Microwave Conference (RFM), Seremban, Malaysia, pp. 133-136, 2011.

[10] X. Qing, M. Y.-W Chia, and X. Wu, "Wide-slot antenna for UWB applications," IEEE Antennas and Propagation Society International Symposium, vol. 1, pp. 834-837, 2003.

[11] P. J. Soh, G. A. E. Vandenbosch, F. H. Wee, A. van den Bosch, M. Martinez-Vazquez, and D. M. M.-P. Schreurs, "Specific Absorption Rate (SAR) Evaluation of Biomedical Telemetry Textile Antennas", IEEE MTT-S International Microwave Symp. (IMS), Seattle, WA., pp.1-3, 2013. 